Bundesgesundheitsbl 2017 -60:1075-1082 DOI 10.1007/s00103-017-2606-x

Online publiziert: 15. August 2017 (c) Der/die Autor(en) 2017. Dieser Artikel ist eine Open-Access-Publikation.

CrossMark

\author{
Robin Roukens $\mathbf{s}^{1,10} \cdot$ Frank Lauster $^{2,10} \cdot$ Maria Bara ${ }^{3,10} \cdot$ Bernd Eifert $^{4,10}$. \\ Dietrich Willemsen $^{5,10}$. Thomas Randall ${ }^{6,10}$. Jürgen Herzog ${ }^{7,10}$. \\ Constanze Wendt ${ }^{8,10} \cdot$ Tobias Schmidt-Wilcke $^{1,9,10} \cdot$ Stefan Knecht ${ }^{1,9,10}$ \\ 'Mauritius Therapieklinik, Heinrich-Heine Universität Düsseldorf, Meerbusch, Deutschland \\ ${ }^{2}$ Schön Klinik, Bad Aibling, Deutschland \\ ${ }^{3}$ Klinik und Rehabilitationszentrum Lippoldsberg, Wahlsberg, Deutschland \\ ${ }^{4}$ SRH Fachkrankenhaus, Neresheim, Deutschland \\ ${ }^{5}$ DRK-Krankenhaus, Middelburg, Deutschland \\ ${ }^{6}$ Asklepios Kliniken, Schildautal-Seesen, Deutschland \\ ${ }^{7}$ Schön Klinik, München-Schwabing, Deutschland \\ ${ }^{8}$ Labor Dr. Limbach und Kollegen, Heidelberg, Deutschland \\ ${ }^{9}$ Klinische Neurowissenschaften und Medizinische Psychologie, Universitätsklinikum Düsseldorf, \\ Düsseldorf, Deutschland \\ ${ }^{10}$ Für: Hygiene-Kommission der Deutschen Gesellschaft für Neurorehabilitation (DGNR), Meerbusch, \\ Deutschland
}

\title{
Mehrkosten durch multiresistente Erreger in der Neurorehabilitation
}

Diese Maßnahmen benötigen jedoch erhebliche zusätzliche Ressourcen.

Die Zunahme an Patienten mit MRE in Akutkrankenhäusern führt auch zu einer vermehrten Nachfrage nach Rehabilitationsplätzen für diese Patienten. Dieses Problem trifft besonders die Neurorehabilitation. Sie hat eine Sonderstellung unter den Rehabilitationsdisziplinen. Das neurorehabilitative Phasenmodell erlaubt ihr im Gegensatz zu anderen Disziplinen, Patienten unterschiedlicher Erkrankungsschwere zu versorgen, einschließlich noch kritisch kranker Patienten mit längeren Liegezeiten und intensiveren Kontakten mit Pflege und Therapeuten in der sogenannten Phase B [3]. Damit erfüllt sie eine zentrale Versorgungsfunktion [4], ist aber gleichzeitig besonders mit MRE bei noch kritisch kranken Patienten konfrontiert $[5,6]$.

Im Krankenhaus können MRE-Isolationen in Form eines Operationenund Prozedurenschlüssels (OPS 8-987 - Komplexbehandlung bei Besiedelung oder Infektion mit MRE) berücksichtigt werden. Weil dieser in der Vergangenheit keine hinreichende Erlöswirkung hatte, haben die Gesellschaften für Inne- re Medizin, Infektiologie, Nephrologie, Neurologie und Neurorehabilitation im Jahr 2015 und erneut 2017 dem Institut bezüglich des Entgeltsystems im Krankenhaus einen Vorschlag zur Formulierung eines Zusatzentgelts in Anlehnung an den OPS-Code 8-987 vorgelegt. Auch im ambulanten Bereich fordern Kassenärztliche Vereinigungen bessere Rahmenbedingungen für die Versorgung von Patienten mit MRE [7].

In dem mehrheitlich Tagessatz-basierten Entgeltsystem der Rehabilitation finden MRE-assoziierte Mehrkosten bisher keine einheitliche Berücksichtigung. Rehabilitationskliniken haben sich zu diesem Problem noch nicht in koordinierter Weise positioniert. Brisant wird die Situation dadurch, dass Rehabilitationskliniken im Gegensatz zu Akutversorgungskrankenhäusern Patienten elektiv aufnehmen und meist im Vorfeld wissen, ob zur Aufnahme anstehende Patienten wegen MRE isolationspflichtig sind. Sie können daher bis zu einem gewissen Grad steuern, ob oder in welchem Umfang sie MRE-besiedelte Patienten versorgen. In der Praxis lassen sich infolge der finanziellen Nichtbe- 
rücksichtigung und steigender MREFallzahlen (z.B. Anstieg an MRGN) zwischen den Jahren 2001 und 2009 von 0,41 auf 2,58 pro 1000 Patiententage [2] unterschiedliche Steuerungsmechanismen erkennen. Diese reichen von nachrangiger oder verzögerter Aufnahme bis zur schlichten Ablehnung von betroffenen Patienten.

Diese Praxis ist für betroffene Patienten nachteilig, weil der Rehabilitationserfolg von einem schnellen Beginn abhängt und eine mangelnde Mobilisierung Komplikationen wie Infektionen, Dekonditionierung und Muskelatrophie und damit einen weiteren Funktionsverlust bedingt $[8,9]$. Sie führt aber auch zu einer Verlängerung der Liegezeit und damit zur Kostensteigerung in den Akuthäusern. Nicht zuletzt erhöht der Verbleib dieser Patienten in den Akutkliniken dort die Dichte von MRE-Trägern und somit die Gefahr einer Übertragung auf infektionsgefährdete Schwerstkranke. Zu Beginn des Jahres 2015 kam es im Zusammenhang mit dem 4-MRGN-Keim Acinetobacter baumanii zu Todesfällen im Universitätsklinikum Kiel. Verantwortliche wiesen nachbehandelnden bzw. nicht nachbehandelnden Rehabilitationskliniken eine Teilschuld an den Versorgungsschwierigkeiten des Klinikums zu. Der Sprecher des Universitätsklinikums Schleswig-Holstein (UKSH) formulierte gegenüber der Deutschen Presse-Agentur [10]: „Wir haben Hinweise, dass sich neun Einrichtungen verweigern oder ein Screening - eine Untersuchung über Keime zur Bedingung [der Übernahme - Anmerkung der Verfasser] machen“.

Restriktive Aufnahmen MRE-besiedelter Patienten durch Rehabilitationskliniken sind darin begründet, dass Anforderungen der Hygiene mit dem Ziel der Rehabilitation in Konflikt stehen können. Insbesondere haben Patienten durch Funktionstherapien erheblich mehr und engere Personenkontakte als im Akutkrankenhaus. Patienten benutzen Geräte und Trainingsroboter, oft in kleinen Gruppen oder unmittelbar nacheinander. Und sie sollten zur größtmöglichen Mobilisation das Zimmer verlassen.

Daher bedarf es aufwendiger und umsichtiger Lösungen mit entsprechend hohem Koordinations- und Erläuterungs- bedarf. Mangels entgeltlicher Abbildung kann der isolationsassoziierte Mehraufwand aber bisher nur durch Kürzungen genuiner Rehabilitationsleistungen für andere Patienten erbracht werden.

Daher kann eine erhebliche Steuerungsverzerrung in der Versorgungskette von noch nicht wieder selbstständigen Patienten mit MRE konstatiert werden. An dieser Verzerrung ist weder den Leistungserbringern noch den Patienten gelegen. Für eine fruchtbare Diskussion der Beteiligten über diese Steuerungsverzerrung muss das Problem beschrieben und quantifiziert werden. Die Deutsche Gesellschaft für Neurorehabilitation (DGNR) hat daher ihre Hygienekommission beauftragt, repräsentative Daten zu den Mehraufwendungen für die Versorgung von isolationspflichtigen Patienten zu erheben, die im Folgenden vorgestellt werden.

\section{Methoden}

\section{Verfahren und Teilnehmer der bundesweiten Erhebung}

Zunächst wurden in 4 Treffen mit einer Expertin für Krankenhaushygiene und leitenden Ärzten von neurologisch-neurochirurgischen Rehabilitationseinrichtungen im Rahmen der Hygienekommission der DGNR in den Jahren 2014 und 2015 - angelehnt an ein DelphiVerfahren - Fakten und Probleme zu multiresistenten Erregern zusammengetragen, diskutiert und bewertet. Unter den besprochenen Themen kristallisierten sich die bisher fehlende Vergütung der Versorgung einer zunehmenden Anzahl MRE-isolierter (MRSA, 3MRGN, 4MRGN) Patienten und die Bedeutung bzw. der Einfluss auf die Neurorehabilitation als entscheidend heraus [11]. Es wurde in der Hygienekommission der DGNR beschlossen, eine eigene Datenerhebung zur Kalkulation der Kosten durchzuführen. Hierzu wurde entschieden, eine bundesweite Erhebung mithilfe eines Fragebogens (siehe Anhang) durchzuführen.

Der in einem mehrstufigen Verfahren abgestimmte Fragebogen wurde an 12 in der DGNR-Hygienekommission vertretene Einrichtungen der neurologisch-neurochirurgischen Rehabilitation versandt. An der Erhebung beteiligten sich 7 Rehabilitationskliniken; die übrigen Kliniken beteiligten sich aus organisatorischen oder administrativen Gründen nicht. Die durchgeführten Datenerhebungen erfolgten zwischen Juli 2014 und Mai 2015 auf den Stationen der neurologisch-neurochirurgischen (Früh-)Rehabilitation von 7 Kliniken aus Bayern, Baden-Württemberg, Hessen, Niedersachsen, Nordrhein-Westfalen und Schleswig-Holstein. In insgesamt 741 Betten wurden dort Patienten der neurologisch-neurochirurgischen Rehabilitation vorwiegend der Phasen $\mathrm{B}$ und $\mathrm{C}$ betreut.

Die Ergebnisse der Erhebung wurden in einer weiteren Sitzung der Hygienekommission diskutiert und bewertet. Basierend auf den Ergebnissen des Fragebogens erfolgte die von uns durchgeführte Kostenkalkulation unterteilt in direkte Kosten (Personal, Sachkosten und andere Kosten) und indirekte Kosten (Opportunitäts- also Verzichtskosten).

Die Verteilung der verschiedenen MRE in den Kliniken wurde nicht erfasst.

Der Fragebogen und die Details der indirekten Kostenberechnung sind als ergänzendes Material über den korrespondierenden Autor erhältlich.

\section{Direkte Kosten}

Erfasst wurden die direkten Kosten (Sachkosten, Personalkosten und andere Kosten), z. B. bei MRE-Isolationsschleusen als gut messbarer Kostenfaktor. Hierbei wurde die Anzahl der Kontakte nach Berufsgruppe im Isolationszimmer erfasst und mit der durchschnittlichen Dauer der Schleusenumkleidezeiten multipliziert, um die Personalkosten zu ermitteln. Zudem wurden die Materialkosten im Schleusenbereich ermittelt, anhand der Verbrauchswerte multipliziert mit den Einkaufskosten.

Wir erfassten die Anzahl der Personalkontakte bei MRE (Strichliste an der Zimmertür) sowie den zeitlichen Aufwand zur Vorbereitung, u. a. zum Anziehen der Isolationskleidung. Zur Messung des zeitlichen Aufwands wurden Stoppuhren verwendet. 
Bundesgesundheitsbl 2017·60:1075-1082 DOI 10.1007/s00103-017-2606-x

(c) Der/die Autor(en) 2017. Dieser Artikel ist eine Open-Access-Publikation.

\section{R. Roukens · F. Lauster $\cdot$ M. Bara · B. Eifert · D. Willemsen · T. Randall $\cdot$ J. Herzog $\cdot$ C. Wendt $\cdot$ T. Schmidt-Wilcke $\cdot$ S. Knecht} Mehrkosten durch multiresistente Erreger in der Neurorehabilitation

\section{Zusammenfassung}

Hintergrund. Die Zahl von Patienten mit multiresistenten Erregern (MRE) in Rehabilitationseinrichtungen nimmt zu. Der dadurch steigende hygienemedizinische Mehraufwand mindert Ressourcen für rehabilitative Kernleistungen. Neben der so entstehenden Fehlversorgung besteht ein weiteres Risiko darin, dass MRE-tragende Patienten nur noch nachrangig in Rehabilitationseinrichtungen aufgenommen werden. Die Hygienekommission der Deutschen Gesellschaft für Neurorehabilitation (DGNR) unternahm daher nachfolgende Abschätzung des Fehlversorgungsrisikos.

Methoden. Zur Analyse der Mehrkosten für die Rehabilitation von Patienten mit MRE wurden in der Hygienekommission der DGNR Kriterien für eine Aufwandserfassung identifiziert. Direkte (Sach- und Personalkosten sowie sonstige Kosten) und indirekte Kosten durch Opportunitätsverluste wurden in 7 Rehabilitationskliniken in unterschiedlichen Bundesländern erfasst.

Ergebnisse. Im Mittel ergaben sich für hygienemedizinische Isolationsmaßnahmen pro Tag direkte Kosten in Höhe von $144 €$ (47 € Sachkosten, $92 €$ Personalkosten und $5 €$ andere Kosten wie z. B. Transportmehraufwendungen) sowie indirekte Kosten von $274 €$. Somit errechneten sich tägliche Zusatzkosten von $418 €$ pro MRE-besiedeltem Patienten. Angesichts einer Besiedlungsrate von $10 \%$ bindet dieser Mehraufwand annähernd ein Zehntel des Gesamtbudgets der Rehabilitationseinrichtungen und wird mit Zunahme von MRE-Besiedlungszahlen weiter steigen.

Schlussfolgerung. Die Aufnahme von Patienten mit MRE in Neurorehabilitationskliniken verursacht Mehrkosten in einem Ausmaß, das die Fähigkeit der Kliniken zur rehabilitativen Kernleistung kritisch beeinträchtigt.

Schlüsselwörter

Antibiotikaresistenz $\cdot$ Isolationsmaßnahmen . Rehabilitation · Neurorehabilitation . Gesundheitsökonomie

\section{Costs associated with multiresistant bacteria in neurorehabilitation}

\section{Abstract}

Background and Objectives. The number of patients with multiresistant bacteria (MRB) in rehabilitation facilities is increasing. The increasing costs of hygienic isolation measures reduce resources available for core rehabilitation services. In addition to the existing lack of care, patients with MRB are at further risk of being given lower priority for admission to rehabilitation facilities. Therefore, the Hygiene Commission of the German Society for Neurorehabilitation (DGNR) attempted to quantify the overall risk for deterioration of rehabilitation care due to the financial burden of MRB.

Materials and Methods. To analyze the added costs associated with the rehabilitation of patients with MBR, the DGNR Hygiene Commission identified criteria for a cost assessment. Direct (consumables, personnel and miscellaneous costs) and indirect costs of loss of opportunity were evaluated in seven neurorehabilitation centers in different states across Germany.

Results. On average, hygienic isolation measures amounted to direct costs of $144 €$ per day ( $47 €$ consumables, $92 €$ personnel, $5 €$ for other costs such as extra transportation expenditure) and indirect costs of $274 €$, totaling $418 €$ per patient with MRB per day. Given that approximately $10 \%$ of patients had $M R B$, the added costs of hygienic isolation measures equaled about one tenth of the overall budget of a rehabilitation center and can be expected to rise with the increasing numbers of patients with MRB.

Conclusions. Admission of patients carrying MRB to neurorehabilitation centers triggers added costs that critically diminish the overall capacity for centers to provide their core rehabilitation services.

\section{Keywords}

Antibiotic resistance $\cdot$ Isolation measures . Rehabilitation · Neurorehabilitation $\cdot$ Health economy
Ergänzend wurden die Materialkosten anhand des kalkulierten Mindestverbrauchs ermittelt. Die Daten wurden vom Einkauf der Kliniken bereitgestellt. Vom Controlling der Kliniken wurden die Personaldurchschnittslohnkosten sowie die Anzahl der MRE-Isolierungen pro Tag und im Jahresdurchschnitt ermittelt und eingebracht.

Ein weiterer ermittelter Kostenfaktor waren die Aufwendungen für Eradikationsmaßnahmen (nur MRSA), hier wurden die Gesamtkosten pro Jahr über die Apotheken ermittelt. Eine gesonderte MRE-Zimmerschlussdesinfektion wurde nicht berücksichtigt. Ergänzend wurden die Isolationsfahrten, z. B. zur externen konsiliarischen Diagnostik, erfasst.

Direkte Kosten des Mehraufwands durch therapeutische Maßnahmen im Patientenzimmer, Sanierungsmaßnahmen usw. wurden anhand der im DRGBereich/OPS 8-987 verwendeten Kennzahlen berechnet, unabhängig von der Phasenzuordnung der Rehabilitation.

Weiter wurde ein zusätzlicher Kostenblock für Verlaufsscreenings und erweiterte mikrobiologische Diagnostik, z. B. nach Sanierungsmaßnahmen, und erhöhten täglichen Mehraufwand, u. a. für die Aufklärung wechselnder Angehöriger und Besucher sowie die Koordination von Pflege, Therapeuten, Ärzten und medizinisch-technischen Assistenten, geschätzt.

Nicht erfasst wurden Eingangsscreeningkosten, Kosten für Fachkräfte und Schulungen sowie Kosten des diagnostischen Mehraufwands, z. B. Schutzhüllen für Sonographiegeräte, Isolationstransporte im Haus, Probleme im Fall von Verlegungsnotwendigkeit bei akuter Erkrankung und MRE usw., da es sich hier 


\begin{tabular}{|c|c|c|c|c|c|c|}
\hline & Schleuszeit & $\begin{array}{l}\text { Anzahl der Iso- } \\
\text { lationskontakte } \\
\text { pro Tag }\end{array}$ & $\begin{array}{l}\text { Zeitlicher the- } \\
\text { rapeutischer } \\
\text { Mehraufwand }\end{array}$ & $\begin{array}{l}\text { Materieller the- } \\
\text { rapeutischer. } \\
\text { Mehraufwand }\end{array}$ & $\begin{array}{l}\text { Sanierungs- } \\
\text { kosten }\end{array}$ & $\begin{array}{l}\text { Anzahl der Isola- } \\
\text { tionsfahrten pro } \\
\text { Monat }\end{array}$ \\
\hline & (s) & (n) & ( $\epsilon /$ Tag) & ( $\epsilon /$ Tag) & (€/Jahr) & (n) \\
\hline Klinik 1 & 60 & 30 & 40 & 21 & 15.484 & 25 \\
\hline Klinik 2 & 60 & 35 & 60 & k. A. & 13.700 & 25 \\
\hline Klinik 3 & 65 & 30 & 150 & k. A. & k. A. & k. A. \\
\hline Klinik 4 & 90 & 33 & 60 & 24,75 & 1500 & 2 \\
\hline Klinik 5 & 90 & 30 & 50 & 10 & 3559 & 30 \\
\hline Klinik 6 & 115 & k. A. & 43 & 8,5 & 6200 & 0 \\
\hline Klinik 7 & 78 & 26 & 101 & 36 & k. A. & 0 \\
\hline Mittelwert & 80 & 30 & 72 & 20 & 8088 & 14 \\
\hline Standardabweichung & \pm 20 & \pm 3 & \pm 40 & \pm 11 & \pm 6198 & \pm 14 \\
\hline
\end{tabular}

Tab. 2 Personalkosten (gerundet) resultierend aus zeitlichem Mehraufwand und Bruttolohnkosten von $45 € / h$ entsprechend Erhebung in Klinik in Nordrhein-Westfalen

Direkte Kosten: Personal (pro Tag pro Patient)

Schleuse

Aufklärung

Koordination

Therapeutischer Mehraufwand

Summe

um regulär geforderte Hygienemaßnahmen handelt.

Ebenso nicht erfasst wurden auf die Rehabilitation wirkende Effekte wie affektive Störungen und Anpassungsstörungen durch Isolation und Minderung der Therapiedichte durch Hygienemaßnahmen sowie Wegfall von Gruppentherapien, d.h. Auswirkungen, die wahrscheinlich die Rehabilitationsdauer und -fortschritte beeinflussen.

\section{Ergebnisse}

\section{Direkte Kosten - Personalkosten}

\section{Schleuse}

Die Messungen ergaben einen Zeitaufwand von im Durchschnitt $80 \mathrm{~s}$ zum Anlegen der Schutzkleidung (Mundschutz, Handschuhe und Schutzkittel) und wurden beim therapeutischen, ärztlichen, pflegerischen und Servicepersonal erhoben. Der Ablauf des Umkleidens war nicht einheitlich vorgegeben. Die Ausschleuszeit wurde mit $30 \%$ der gemessenen Einschleuszeit berechnet.

Bei durchschnittlich 30 Kontakten pro Tag in allen Kliniken (beispielhaft die Klinik in Nordrhein-Westfalen: 7 the- rapeutische Kontakte, 2 Arztkontakte, 3 Servicepersonalkontakte, 18 pflegerische Kontakte) ergibt sich ein zeitlicher Mehraufwand von 52 min pro Tag pro Patient. Hierbei sind die pflegerischen und therapeutischen Kontakte mit über $80 \%$ der Kontakte in der Mehrzahl (s. - Tab. 1).

Bei durchschnittlichen Stundenarbeitskosten (gemittelt aus ärztlichen und nicht ärztlichen Arbeitskosten, Bruttoarbeitslohn plus Lohnnebenkosten) von $45 €$ in Rehabilitationskliniken ergeben sich bei 52 min Zeitaufwand Arbeitskosten der Isolation in Höhe von $39 €$ pro Tag (s. • Tab. 2).

\section{Aufklärung}

Für jeden Isolierungsfall kann zudem mit einer Aufklärungszeit (Patient, Angehörige, Mitpatienten, wechselnde Besucher u. a.) von $15 \mathrm{~min}$ pro Tag, verteilt auf unterschiedliche Mitarbeiter, gerechnet werden. Je nach Fall weichen diese Zahlen deutlich nach oben ab. In Übereinstimmung aller beteiligten Kliniken wurde insbesondere im Vergleich zu höheren Werten in der DRG-Kalkulation - mit diesem niedrigen Wert gerechnet. Kosten durch Aufklärung können hier mit
$11,25 €$ pro Isolationspatient pro Tag berechnet werden $(0,25 \mathrm{~h}$ bei Arbeitskosten von $45 €$ pro Stunde). Da der Aufklärungsaufwand im Verlauf der Behandlung unterschiedlich verteilt ist, am Anfang größer und gegen Ende weniger, wurde der Aufklärungsaufwand pauschal um $50 \%$ nach unten korrigiert auf 5,6€.

\section{Hygienekoordinationsaufwand}

Für die Hygienemaßnahmen ist ein erhöhter Koordinationsaufwand nötig. 15 min pro Tag werden veranschlagt für Absprachen der Hygieneabteilung mit Ärzten, Therapieplanung, Funktionstherapeuten, Pflege und medizinischtechnischen Assistenten und für $\mathrm{Ab}$ sprachen dieser Berufsgruppen untereinander. Da hier jeweils wenigstens 2 Mitarbeiter eingebunden sind, kalkulieren sich 30 Mitarbeiterminuten bei gemischter Stundenlohnannahme Ärzte/nichtärztliches Personal von $45 €$ pro Stunde mit 22,5€ pro Tag. Wegen des hier ebenfalls sich im Verlauf der Behandlung ändernden Koordinationsaufwands korrigierten wir auch diesen Posten um $50 \%$ nach unten auf $11,25 €$. Dies ist ebenfalls im Vergleich mit der DRGKalkulation eine konservative Berechnung. Durch eine klare Organisation der Abläufe kann eine Reduktion auf ein Minimum notwendiger Absprachen erreicht werden; hierbei sind auch die Optimierung der Informationstechnologie (IT) und die klare Kennzeichnung von MRE-Patienten und MRE-Zimmern zu nennen. 
Tab. 3 Sachkosten (gerundet) resultie-

rend aus Kosten für Verbrauchsmaterialien

und für die Therapien unter Isolationsbedin-

gungen angefertigten Materialien, z. B. im

Bereich der Ergotherapie und der Neuropsy-

chologie

Direkte Kosten: Sachkosten (pro

(€)

Tag pro Patient)

Schleuse 11

Sanierung

2

Verlaufsscreening

14

Therapeutischer Mehraufwand

Summe

20

47

\section{Therapeutischer Mehraufwand}

Für den per Delphi-Verfahren ermittelten zeitlichen therapeutischen Mehraufwand über die Schleusenzeiten hinaus (Vorbereitung und Nachbereitung von Materialien und Geräten wie z. B. Stehtrainer und Ergometer in der Physiotherapie und Arbeits- bzw. Therapiematerial in Logopädie, Ergotherapie und Neuropsychologie für jeweils 4-6 Therapieeinheiten unter Isolationsbedingungen) ergeben sich bei $30 €$ Stundenlohn multipliziert mit $72 \mathrm{~min}$ (entsprechend 1,2 h) Mehrkosten in Höhe von $36 €$ pro Tag. Auch hier liegen die Werte unter denen der DRG-Kalkulation (• Tab. 2).

\section{Direkte Kosten - Sachkosten}

\section{Schleuse}

Pro Kontakt wurden durchschnittlich 2 Handschuhe (4,6 Cent, Beispiel Nordrhein-Westfalen), ein Schutzkittel (26,5 Cent, Beispiel Nordrhein-Westfalen) sowie eine Maske (5,15 Cent, Beispiel Nordrhein-Westfalen) berechnet. Hieraus ergeben sich Kosten von 36,25 Cent pro Umkleidevorgang und bei 30 Isolationszimmerkontakten Kosten von $11 €$ pro Tag.

\section{MRSA-Sanierungskosten}

Die MRSA-Sanierungskosten (u. a. Nasensalbe und Mundspray) betragen $8090 €$ pro Jahr für die Durchschnittsklinik (106 Betten/10,1 \% Isolation) und somit $2 €$ pro Isolationstag.

\begin{tabular}{|c|c|c|c|c|}
\hline & \multirow{2}{*}{$\begin{array}{l}\text { Anzahl der } \\
\text { Betten } \\
\text { (n) }\end{array}$} & \multirow{2}{*}{$\begin{array}{l}\text { Isolationsquote } \\
\text { (\%) }\end{array}$} & \multirow{2}{*}{$\begin{array}{l}\text { Einbettzimmer } \\
\text { (\%) }\end{array}$} & \multirow[t]{2}{*}{$\begin{array}{l}\text { Höhe der } \\
\text { Tagessätze }\end{array}$} \\
\hline & & & & \\
\hline Klinik 1 & 157 & 10,2 & 10 & IGES \\
\hline Klinik 2 & 150 & 12 & 17 & DRG \\
\hline Klinik 3 & 50 & 11 & 12 & $>$ IGES \\
\hline Klinik 4 & 87 & 4,3 & 17,5 & IGES \\
\hline Klinik 5 & 200 & 10 & 1 & IGES \\
\hline Klinik 6 & 42 & 4,9 & 18 & IGES \\
\hline Klinik 7 & 55 & 17,4 & 6 & IGES \\
\hline Mittelwert & 106 & 10 & 12 & IGES \\
\hline Standardabweichung & $\pm 62,7$ & $\pm 4,4$ & \pm 6 & - \\
\hline
\end{tabular}

\section{Verlaufsscreening und Koordinationsaufwand}

Die Kosten für Verlaufsscreenings beliefen sich entsprechend der durchgeführten Prozeduren und Jahresrechnungsstellung beispielhaft in einer Klinik in Nordrhein-Westfalen auf $14 €$ pro Tag. Die Anzahl der Screenings ist hierbei abhängig von der Art des MRE (Abstriche und/ oder Kulturen); die durchschnittlichen Kosten wurden durch die ControllingAbteilungen ermittelt.

\section{Therapeutischer Mehraufwand}

Die geschätzten direkten Kosten für den therapeutischen Mehraufwand für zusätzliche Materialien (für die Therapie unter Isolationsbedingungen angeschaffte Einmalartikel oder speziell erstellte Therapiematerialien, die nicht mehr aus dem Isolationszimmer entfernt werden, insbesondere in der Bereichen Ergotherapie und Neuropsychologie) betragen $20 €$ pro Tag (• Tab. 3).

\section{Direkte Kosten - andere Kosten}

\section{Transportkosten}

Die Kosten für Isolationstransporte (z. B. zur radiologischen Diagnostik oder zu Konsiluntersuchungen in $u$. a. Urologie und Neurochirurgie) betragen bei durchschnittlich 14 Transporten je Klinik pro Monat und der Differenz zwischen regulärem Krankentransport und Isolationsfahrt von $120 €$ bei jedoch großer regionaler Schwankungsbreite entsprechend $1680 €$ pro Monat, also $56 €$ pro Tag. Da- raus ergeben sich bei 106 Betten und $10 \%$ Isolationsquote (für die Durchschnittsklinik) Kosten von 5,16€ pro Tag pro Isolationsbett.

\section{Indirekte Kosten}

\section{Opportunitätskosten}

In der errechneten Durchschnittsklinik ist die Anzahl der MRE-Fälle mit Isolationspflichtigkeit größer als die verfügbare Anzahl an Einzelzimmern (EZ; - Tab.4). Daher erfolgt die Berechnung der Opportunitätskosten (OP) durch nicht belegte Betten nach der Formel OP $=\mathrm{C} \times \mathrm{TP} \times$ $\left(\mathrm{MRE}_{\mathrm{EZ}}\right.$ - EZ-Kapazität)/MRE (eine Erläuterung der Berechnung erfolgt im supplementären Material, zu beziehen über den Korrespondenzautor). Als Tagespauschale (TP) wird der unterste Wert des IGES-Mittelwertbereichs (IGES Institut GmbH-Mittelwertbereich Phase B: $450-550 €)$ verwendet. Der Korrekturfaktor $\mathrm{C}$ ist geschätzt und soll berücksichtigen, dass bei einem leerstehenden Bett ein Teil der Kosten z. B. für Medikamente und Ernährung nicht anfallen. Daraus ergibt sich: $\mathrm{OP}=0,75$ (Korrekturfaktor) $\times 450 € \times 10,7$ (MREisolationspflichtige Betten entsprechend $10,1 \%$ von 106 Betten) - 2 (Anzahl Betten EZ-Kapazität entsprechend 12,7 Einzelzimmerbetten abzüglich 10,7 Betten $10 \%$ iger Planungsmarge bei fehlenden nicht belegten Betten)/10,7 (MRE-Fälle); $\mathrm{OP}=0,75 \times 450 € \times[(10,7-2) / 10,7]=$ $(0,75 \times 450 \times 0,813) €=274 €$. 


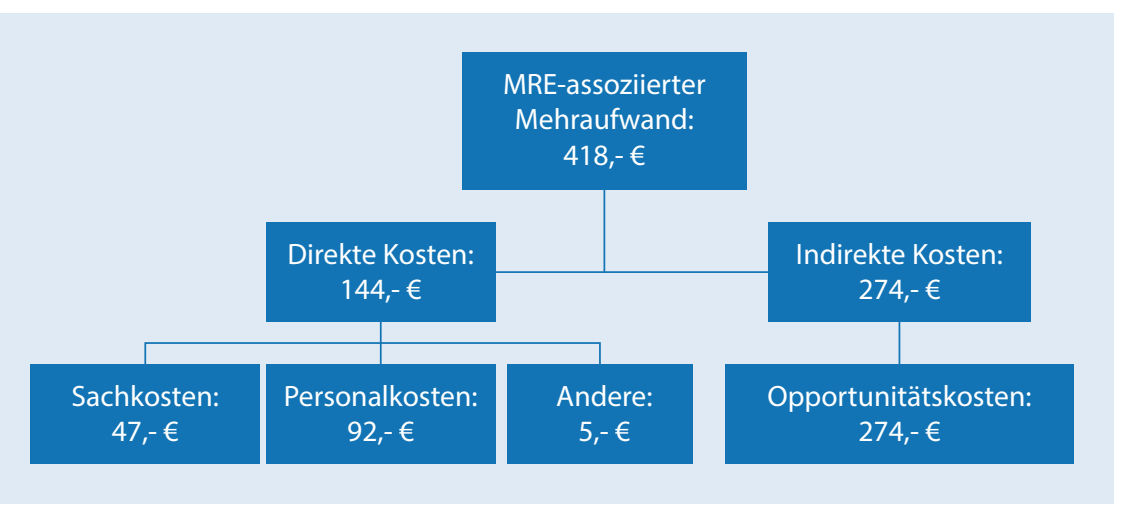

Abb. 1 A Aufteilung des Mehraufwands für die Versorgung von mit multiresistenten Erregern (MRE) besiedelten Patienten in der Neurorehabilitation der Phase B (Kosten auf volle Eurobeträge gerundet)

Damit liegen die Opportunitätskosten im Modell des vorherrschend von der MRE-Problematik betroffenen Bereichs der Neurorehabilitationsphase $B$ bei $274 €$ pro Tag (• Abb. 1). In der Neurorehabilitationsphase C belaufen sich die Opportunitätskosten bei gleichen Grundannahmen für Patienten mit MRE auf $137 €$ pro Tag.

\section{Diskussion}

Hauptergebnis der vorliegenden Erhebung ist, dass in der stationären Neurorehabilitation der Phase B die Versorgung eines Patienten, der mit einem MRE besiedelt ist, zusätzliche Kosten von $418 €$ pro Tag verursacht (in der Phase B grundsätzlich Einzelzimmerisolation bei MRE entsprechend den Richtlinien des Robert Koch-Instituts bzw. den Empfehlungen der Kommission für Krankenhaushygiene und Infektionsprävention).

Dieser Mehraufwand resultiert einerseits aus direkten Kosten (144€ pro Tag). Diese ergeben sich aus zusätzlichem Aufwand für Personal und Sachmittel sowie Kosten für gelegentliche Krankentransporte unter erhöhtem Isolationsaufwand.

Andererseits resultiert der Mehraufwand aus indirekten Kosten (274€). Diese werden dadurch verursacht, dass in Zimmern mit isolierten Patienten meist keine anderen Patienten versorgt werden können.

Für Akutkliniken im DRG-Bereich in Deutschland berechneten Hübner et al. [13] MRSA-assoziierte Kosten von über $8600 €$ pro Fall (bei 17 Isolationstagen entspricht dies $506 €$ pro Tag) bei mit
77,45 \% ebenfalls hohem Anteil an Opportunitätskosten.

Die hoch erscheinenden Kosten und der hoch erscheinende zeitliche Aufwand bei einigen direkten Kosten sind auf Schätzungen basiert und wurden in Anlehnung an ein Delphi-Verfahren ermittelt. Es stellte sich heraus, dass die ärztlichen Mitarbeiter den zeitlichen und materiellen Aufwand deutlich unterschätzten.

Bei Gesprächen zur Arbeit mit MRE und Beobachtungen des Vorgehens der nicht ärztlichen Mitarbeiter in den unterschiedlichen Bereichen wurde deutlich, dass ein erheblicher und wiederkehrender Aufwand entsteht. Eine veränderte Risikostratifizierung im Verlauf, z. B. in Abhängigkeit von Monitornotwendigkeit, Kooperationsfähigkeit und unterschiedlichen keimbezogenen Hygienekonzepten, führt $\mathrm{zu}$ wiederkehrenden und zeitintensiven Absprachen verschiedener Berufsgruppen. Auch müssen dieselben Angehörigen teilweise pro Schicht wiederholt erinnert und in Hygienemaßnahmen eingewiesen werden. Das Gleiche gilt für den Materialaufwand sowohl therapeutisch als auch pflegerisch.

Wir möchten betonen, dass die von uns erhobenen Werte niedriger sind, als die der DRG-Kalkulation zugrunde liegenden Aufwendungen und wir um eine konservative Vorgehensweise bemüht waren. Auch die Kalkulationen aus dem Akutkrankenhausbereich fallen mit Gesamttageskosten von über $500 €$ höher aus.
Da es bisher keine vergleichbaren Erhebungen aus dem Bereich der Rehabilitation gibt, fällt eine Einordnung schwer und wird erst durch spätere vergleichbare Kalkulationen möglich.

Der Korrekturfaktor zur Berechnung der Opportunitätskosten erscheint uns eine plausible betriebswirtschaftliche Handhabung bei einer hohen Personalkostenquote und geringen variablen Kosten im Bereich der Rehabilitation.

Unserer Erhebung lagen die aktualisierten KRINKO-Empfehlungen zur Prävention und Kontrolle von MRE in medizinischen und pflegerischen Einrichtungen zugrunde.

Diese Ergebnisse fußen auf einer breiten Datenbasis von insgesamt $741 \mathrm{Neu}$ rorehabilitationsbetten in 7 Neurorehabilitationskliniken in 6 Bundesländern. Hier lag die mittlere Isolationsquote bei $10 \%$ der belegbaren Betten. Die MRE-assoziierten Mehraufwendungen entsprechen einem Großteil des Tagessatzes in der Phase B der Neurorehabilitation. Daraus ergibt sich, dass der in den untersuchten Häusern nachgewiesene Mehraufwand für die Versorgung von Patienten mit MRE grob $10 \%$ des Gesamtbudgets der Einrichtungen bindet. Diese Bindung von Ressourcen bedeutet, dass aktuell die genuine neurorehabilitative Leistungsfähigkeit der untersuchten Häuser um ein Zehntel reduziert ist. Eine Zunahme von mit MRE besiedelten Patienten in den Kliniken würde deren rehabilitative Leistungsfähigkeit weiter senken. Bei Fortbestehen der gegebenen Rahmenbedingungen müssten Neurorehabilitationskliniken daher noch restriktiver als bisher mit der Aufnahme betroffener Patienten umgehen, wenn sie ihre Kernleistungsfähigkeit nicht verlieren wollen.

Insgesamt bemisst die vorliegende Erhebung in Zahlen das Dilemma zwischen der Häufung von MRE und dem Verlegungsbegehren durch Krankenhäuser einerseits und der Gefährdung der rehabilitativen Leistungsfähigkeit von Neurorehabilitationskliniken andererseits. Vonseiten der Rehabilitationskliniken kann bei ausbleibender Finanzierung diesem Dilemma aktuell nur durch Aufnahmerestriktionen begegnet werden. Ein fehlender oder erschwerter Rehabilitationszugang für mit MRE besiedelte 
Patienten reduziert jedoch deren Chancen auf Wiederherstellung von Unabhängigkeit [4], steigert ihr Mortalitätsrisiko [9] und vermehrt die Nettokosten in der Versorgungskette [12]. Ferner belasten zusätzliche Behinderungen des Zugangs zur poststationären Rehabilitation die Krankenhäuser weiter und damit die Akutversorgung im Land.

Weitere Untersuchungen sind notwendig, um auch die Mehrkosten durch MRE in anderen Rehabilitationsphasen zu ermitteln.

\section{Limitationen}

In dieser Arbeit haben wir Daten aus einer begrenzten Zahl von Kliniken aus mehreren Bundesländern erhoben und gemittelt. Wegen unterschiedlicher Versorgungsstrukturen in den Bundesländern und betriebswirtschaftlichen Unterschieden zwischen Kliniken können sich MRE-assoziierte Kosten zwischen Häusern unterscheiden. Nichtsdestotrotz existieren aktuell unseres Wissens keine genaueren und umfassenderen Erhebungen. Opportunitätskosten und deren große Bedeutung für die Gesamtkosten sind stark beeinflusst durch lokale Faktoren, entsprechen jedoch den Ergebnissen anderer Datenerhebungen [13]. Ferner können unter sehr eingegrenzten Umständen Patienten mit MRE kohortiert isoliert werden, sodass in diesem Fall Opportunitätskosten entfielen. Dies betrifft aber nur eine Minderzahl von Fällen.

Eine weitere Limitation ist die Tatsache, dass nur ein Teil der direkten Kosten auf überprüfbaren Belegen basiert und für die anderen Bereiche Schätzungen herangezogen werden müssen - dieser Tatsache wurde mit einem über die DGNR koordinierten, an ein Delphi-Verfahren angelehnten Vorgehen Rechnung getragen.

\section{Schlussfolgerung}

Während die Mehraufwendungen für die Hygienemaßnahmen in der Versorgung von neurologisch-neurochirurgischen Rehabilitationspatienten - insbesondere der Phase B mit MRE - heute bereits die Ressourcen für genuine Rehabilita- tionsleistungen um etwa $10 \%$ mindern, wird dieser Anteil mit Zunahme von MRE weiter ansteigen. Wahrscheinlich stehen hinter diesen Zahlen zusätzlich viele Patienten, die wegen ihres MRETrägerstatus nicht oder nur verzögert von Rehabilitationskliniken versorgt wurden.

Aus Sicht der Autoren erscheint der einzig sinnvolle Weg zur Lösung des Problems die Definition eines Zusatzentgelts für die Versorgung von MRE in der neurologisch-neurochirurgischen $\mathrm{Re}$ habilitation. Die vorliegende Erhebung gibt einen Anhaltspunkt für die Kalkulation eines solchen MRE-Zuschlags. Öffentlichkeit, Politik und Kostenträger bekennen sich zur Bekämpfung von MRE - und wichtiger noch - zur adäquaten Behandlung MRE-besiedelter Patienten. Die Ernsthaftigkeit dieses Bekenntnisses wird sich danach bemessen lassen, inwieweit die Leistungserbringer in die Lage versetzt werden, hier ihren Versorgungsauftrag zu erfüllen.

\section{Fazit für die Praxis}

\section{- Besiedlungen mit MRE erfordern} kostenträchtige zusätzliche Hygienemaßnahmen.

- Kosten für Hygienemaßnahmen bei Besiedlung mit MRE resultieren aus personellen und sächlichen Mehraufwendungen und verlorenen Einnahmen durch Nichtbelegbarkeit von Nachbarbetten.

- Mehrkosten durch MRE in der Neurorehabilitation reduzieren die Ressourcen für die eigentliche Rehabilitation.

- Mehrkosten pro Patient und Tag durch MRE in der Neurorehabilitation der Phase B sind annähernd so hoch wie die für die gesamte Behandlung entrichteten Entgelte pro Patient und Tag.

- Der Anteil von Patienten mit MRE ist somit direkt proportional zur reduzierten genuinen Rehabilitationsleistung für alle Patienten und damit der rehabilitativen Effizienz der betroffenen Klinik.

\section{Korrespondenzadresse}

Prof. Dr. med. S. Knecht

Mauritius Therapieklinik, Heinrich-Heine Universität Düsseldorf

Strümper Straße 111, 40670 Meerbusch,

Deutschland

Stefan.Knecht@stmtk.de

\section{Einhaltung ethischer Richtlinien}

Interessenkonflikt. R. Roukens, F. Lauster, M. Bara, B. Eifert, D. Willemsen, T. Randall, J. Herzog, T. SchmidtWilcke und S. Knecht geben an, Angestellte von neurologischen Rehabilitationskliniken zu sein. C. Wendt gibt an, dass kein Interessenkonflikt besteht.

Dieser Beitrag beinhaltet keine von den Autoren durchgeführten Studien an Menschen oder Tieren.

Open Access. Dieser Artikel wird unter der Creative Commons Namensnennung 4.0 International Lizenz (http://creativecommons.org/licenses/by/4.0/deed. de) veröffentlicht, welche die Nutzung, Vervielfältigung, Bearbeitung, Verbreitung und Wiedergabe in jeglichem Medium und Format erlaubt, sofern Sie den/die ursprünglichen Autor(en) und die Quelle ordnungsgemäßnennen, einen Link zur Creative Commons Lizenz beifügen und angeben, ob Änderungen vorgenommen wurden.

\section{Literatur}

1. Geffers C, Gastmeier P (2011) Nosocomial infections and multidrug-resistant organisms in Germany: epidemiological data from KISS (the Hospital Infection Surveillance System). Dtsch Arztebl Int 108:87-93. doi:10.3238/arztebl.2011. 0087

2. Robert Koch-Institut (2015) Zur Entwicklung nosokomialer Infektionen im KrankenhausInfektions-Surveillance-System (KISS). Epidemiol Bull:35-40.doi:10.17886/EpiBull-2015-011.3

3. Knecht $S$, Hesse S, Oster P (2011) Rehabilitation after stroke. Dtsch Arztebl Int 108:600-606. doi:10. 3238/arztebl.2011.0600

4. Knecht $S$, Roßmüller J, Unrath $M$, Stephan KM, Berger K, Studer B (2016) Old benefit as much as young patients with stroke from high-intensity neurorehabilitation: cohort analysis. J Neurol Neurosurg Psychiatr 87:526-530. doi:10.1136/ jnnp-2015-310344

5. Cao J, Min L, Lansing B, Foxman B, Mody L (2016) Multidrug-resistant organisms on patients hands: a missed opportunity. JAMA Intern Med 176(5):705-706. doi:10.1001/jamainternmed. 2016.0142

6. Pohl M, Bertram M, Bucka C et al (2016) Course of rehabilitation in early neurological/neurosurgical rehabilitation. Results of a 2014 multi-center evaluation in Germany. Nervenarzt 87(6):634-644 doi:10.1007/s00115-016-0093-1

7. Deutsches Ärzteblatt (2015) Praxen im Kampf gegen MRSA stärken. https://www.aerzteblatt.de/ nachrichten/63087/Praxen-im-Kampf-gegenMRSA-staerken. Zugegriffen: 10. Juni 2016

8. Gruther W, Benesch T, Zorn C et al (2008) Muscle wasting in intensive care patients: ultrasound observation of the M. quadriceps femoris muscle 
layer. J Rehabil Med 40:185-189. doi:10.2340/ 16501977-0139

9. Scrutinio D, Monitillo V, Guida P et al (2015) Functional gain after inpatient stroke rehabilitation. Stroke 46:2976-2980. doi:10.1161/STROKEAHA. 115.010440

10. Deutsche Presse Agentur (2015) Kieler Keime Klinik-Chef verärgert über Reha-Zentren. https:// www.welt.de/newsticker/dpa_nt/infoline_nt/ brennpunkte_nt/article136819427/Klinik-Chefveraergert-ueber-Reha-Zentren.html. Zugegriffen:27.Jan. 2015

11. Rollnik JD, Samady AM, Grüter L (2014) Multidrug-resistant germs in neurological early rehabilitation (2004-2013). Rehabilitation (Stuttg) 53(5):346-350. doi:10.1055/s-0034-1375640

12. Caro JJ, Huybrechts KF, Kelley HE (2001) Predicting treatment costs after acute Ischemic stroke on the basis of patient characteristics at presentation and early dysfunction. Stroke 32:100-106. doi:10. 1161/01.str.32.1.100

13. Hübner C, Hübner NO, Hopert K, Maletzki S, Flessa S (2014) Analysis of MRSA-attributed costs of hospitalized patients in Germany. Eur J Clin Microbiol Infect Dis 33:1817-1822. doi:10.1007/ s10096-014-2131-x 